\title{
Evaluación de distintos materiales para la elaboración del pykrete y su uso para conservar la cadena de frío
}

\section{Evaluation of different materials for the production of pykrete and its use to preserve the cold chain}

\author{
Daniel Ricardo Delgado ${ }^{1}$, Andrés F. Rivera² ${ }^{2}$ Diego Iván Caviedes Rubio ${ }^{3}$, \\ Germán Escobar Fiesco ${ }^{4}$ y Jhon Jairo Pinzón Barrantes ${ }^{5}$
}

\begin{abstract}
Resumen
El pykrete es un material compuesto por aserrín y agua, cuyas propiedades termofísicas permiten que pueda ser usado como dispositivo refrigerante en cadenas de frío, debido a que no se descongela a la misma velocidad que el hielo y tiene una durabilidad y resistencia mayor. De acuerdo a los resultados de la presente investigación, la relación agua-material celulósico de $14 \% \mathrm{P} / \mathrm{P}$ es la más adecuada puesto que tiene propiedades termodinámicas similares al gel comercial que se utiliza para mantener la cadena de frio. Finalmente el material que se comporta de manera similar al gel comercial es el pykrete de cascarilla de arroz molida.
\end{abstract}

Palabras clave: Pykrete; material celulósico; cadena de frío; propiedades termofísicas.

\begin{abstract}
The pykrete is a material composed of sawdust and water, which thermophysical properties that can allow be your used a cooling device in cold chains, because there is thawed at the same speed as the ice and has a higher strength and durability. According to the results of this research, water-cellulosic materials ratio of $14 \% \mathrm{P} / \mathrm{P}$ is the more appropriate since it has similar thermodynamic properties to commercial gel used to maintain the cold chain. The other hand, material behaves in a similar way to the commercial gel is pykrete ground rice husk.
\end{abstract}

Key words: Pykret; cellulosic material; cold chain; thermophysical properties.

1. Licenciado en Biología y Química, Master en Ciencias Química y Doctor en Ciencias Farmacéuticas, Docente investigador del Programa de Ingeniería Industrial de la Universidad Cooperativa de Colombia Sede Neiva. Calle 11 No. 1 G-31 B, danielr.delgado@campusucc.edu.co

2. Ingeniero Industrial de la Universidad Cooperativa de Colombia - Sede Neiva.

3. Magister en Ecología y Gestión de Ecosistemas Estratégicos. Docente Universidad Cooperativa de Colombia. Programa de Ingeniería Industrial. Grupo de Investigación GRIAUCC, diego.caviedesr@campusucc.edu.co

4. Licenciado en Matemáticas y Física, Master en Ciencias Matemática, Estudiante de Doctorado del Instituto de Matemática e Estatística da Universidade de São Paulo (IME-USP)-Brasil.

5. Licenciado en Química, Estudiante de Doctorado en Química del Instituto de Investigaciones en fisicoquímica de la Universidad de Córdoba - Argentina. 


\section{Introducción}

El principal parámetro para la determinación de la vida media de un producto para tener en cuenta en la cadena de frio es la temperatura (Evans, 1992, James y Evans, 1992, James y Evans, 1992b, Giannakourou y Taoukis, 2003a y Giannakourou y Taoukis, 2003b). Un sistema de aseguramiento de calidad y seguridad moderno debe basarse en la prevención de los fallos de calidad y seguridad a través de monitoreo, registro y control de los parámetros críticos durante el periodo de vida media del producto que incluye la fase de postprocesamiento y se extiende hasta el momento de su uso por parte del consumidor final (Evans et al., 1991, Giannakourou et al., 2001, Dubelaar et al., 2001, Giannakourou y Taoukis, 2002, Kennedy et al., 2005, Koutsoumanis et al., 2002 y Koutsoumanis et al., 2005). Uno de los principales factores al cual se debe presentar atención es el la logística de transporte, almacenamiento y manipulación. Las condiciones de temperatura en la cadena de distribución refrigerada (Broekmeulen, 2001).

Una alternativa es el uso de una tecnología desarrollada durante la segunda guerra mundial denominada Pykreto, el cual es un material compuesto congelado hecho de aproximadamente $14 \%$ aserrín o alguna otra forma de pasta de madera (tal como papel) y $86 \%$ en peso de hielo (de 6 a 1 en peso). Se propuso su uso durante la Segunda Guerra Mundial por Geoffrey Pyke a la Royal Navy británica como un material candidato para hacer un porta aviones enorme, imposible de hundir. El Pykreto tiene algunas propiedades interesantes, especialmente su velocidad de fusión relativamente lento (debido a la baja conductividad térmica), y su muy mejorada resistencia y tenacidad sobre el hielo, lo cual lo hace similar al hormigón.

Estudios realizados por Vasilieva y colaboradores, demostraron que un diez por ciento de aserrín proporciona los mejores valores del comportamiento mecánico del material. Este comportamiento optimizado se refiere a la homogeneidad, la procesabilidad, la tenacidad y la fuerza del pykreto. La resistencia a la compresión de pykreto con $10 \%$ de aserrín puede ser de $12 \mathrm{MPa}$ y la resistencia a la flexión puede ser de 3.7 MPa. Cuando se compara a $3 \mathrm{MPa}$ de hielo y 1.2 MPa, estos valores son aproximadamente 3 veces más altos. La ductilidad de pykreto es incluso 10 veces mejor. Al mejorar la tenacidad a la fractura como se muestra por Kuehn y Nixon (1988) el pykreto permite una mayor deformación de la estructura y reduce rupturas por deformación (Vasilieva et al., 2015). De otro lado el pykreto tiene una tasa de fusión relativamente lenta debido a su baja conductividad térmica (Pronk et al., 2014) mejorando su resistencia al choque térmico y lo que hace de este material un posible candidato para el uso como dispositivo de frío.

\section{Materiales y métodos}

El objetivo principal de la investigación es determinar si el Pykrete puede ser utilizado como dispositivo de refrigeración para conservar la cadena de frío, y determinar la mejor relación agua-aserrín o agua-cascarilla de arroz. En este sentido, el Pykrete es comparado con un gel comercial utilizado como sistema de refrigeración en cajas isotérmicas para mantener la cadena de frio de algunos productos, determinado la capacidad calorífica aparente tanto del pykrete como la del gel comercial, mediante un calorímetro de Joule.

Por tanto se presenta un diseño experimental puro factorial $3 \times 3$ evaluando tres tipos de materiales celulósicos (aserrín, cascarilla de arroz molida y cascarilla de arroz sin moler), y tres composiciones (5, 14 y $20 \%$ ) obteniendo así nueve grupos (tipos de pykrete) evaluando así la influencia de la composición y el tipo de material del pykrete sobre el calor latente de fusión aparente de cada uno de los sistemas (Pykretes).

Finalmente los resultados de cada sistema serán comparados con los datos arrojados por el gel comercial aplicando la prueba $\mathrm{G}$ del test de Cochran (Cochran, 1950) para evaluar la homocedasticidad y así garantizar que las variaciones entre grupos correspondan a las variables independientes (porcentaje y tipo de material celulósico) y no a errores experimentales. Posteriormente se aplicará un ANOVA (Delgado, 2014) para comparar los resultados de cada grupo con los resultados del gel comercial e identificar si hay o no diferencias estadísticamente significativas.

\subsection{Preparación del Pykrete}

En un recipiente de aproximadamente $20 \mathrm{ml}$ se depositaron entre $1.000 \pm 0.001$ a $3.000 \pm 0.001$ gramos de aserrín o cascarilla de arroz y se le adicionó la masa de agua necesaria para preparar pykretes con una fracción másica de material celulósico de $0.05,0.14$ y 0.20 . Este procedimiento se llevó a cabo utilizando una balanza analítica Ohaus Pioner PA313 con una sensibilidad de $\pm 0.001 \mathrm{~g}$. Una vez preparada la mezcla se mantuvo durante $48 \mathrm{~h} \mathrm{a}-5^{\circ} \mathrm{C}$ aproximadamente. 


\subsection{Calibración del calorímetro de Joule}

La calibración del calorímetro es determinante en la exactitud de los resultados. Esta calibración generalmente se hace determinando la temperatura de equilibrio del sistema conformado por el calorímetro y una masa determinada de un estándar conocido como el agua, al adicionar al sistema una masa del estándar a una temperatura diferente a la del sistema. La cantidad de energía que se transfiere desde el sistema a la masa de agua que se adiciona, responde a la siguiente expresión (Levine, 2004):

$$
m_{c} c_{c}\left(T_{2}-T_{f}\right)+m_{2} c_{2}\left(T_{2}-T_{f}\right)=m_{1} c_{1}\left(T_{f}-T_{1}\right) \equiv q \quad \text { Ec. } 1
$$

donde $c_{c}, c_{1 \mathrm{y}} c_{2}$ son constantes $m_{1}$ y $m_{2}$ son las masas de agua a las temperaturas $T_{1}$ y $T_{2}$ respectivamente en donde $T_{1}<T_{2}, m_{c}$ es la masa del calorímetro y $q$ es la cantidad de energía que se transfiere desde el sistema a la masa de agua 1 .

A partir de la ecuación 1 , se puede determinar la capacidad calorífica del calorímetro despejando $c_{c}$ obteniendo la siguiente expresión.

$$
c_{c}=\frac{m_{1} c_{1}\left(T_{f}-T_{1}\right)-m_{2} c_{2}\left(T_{2}-T_{f}\right)}{m_{c}\left(T_{2}-T_{f}\right)} \quad \text { Ec. } 2
$$

\subsection{Determinación del calor latente de fusión aparente del Pykrete y gel comercial}

Una vez se determinó el calor específico del calorímetro, a partir del mismo principio de la ecuación 1, se determina la cantidad de energía que se transfiere del sistema al pykrete una vez se alcance la temperatura en equilibrio. En este proceso, el pykrete inicialmente en estado sólido, se funde liberando al sistema agua y material celulósico por lo que la ecuación 1 se expresaría como:

$$
m_{c} c_{c}\left(T_{2}-T_{f}\right)+m_{2} c_{2}\left(T_{2}-T_{f}\right)=m_{P} c_{P}+m_{W P} c_{W P}\left(T_{f}-0^{\circ} \mathrm{C}\right)+m_{A P} c_{A P}\left(T_{f}-0^{\circ} \mathrm{C}\right) \equiv q \text { Ec. } 3
$$

En donde $m_{W P}$ y $m_{A P}$ son la masa del agua y aserrín o cascarilla de arroz presente en el pykrete respectivamente, $m_{P}$ es la masa de pykrete y $c_{P}$ es el calor latente de fusión aparente del pykrete. Así, a partir de la ecuación $3 c_{p}$ se calcularía como:

$$
c_{P}=\frac{m_{c} c_{c}\left(T_{2}-T_{f}\right)+m_{2} c_{2}\left(T_{2}-T_{f}\right)-m_{W P} c_{W P}\left(T_{f}-0^{\circ} \mathrm{C}\right)-m_{A P} c_{A P}\left(T_{f}-0^{\circ} \mathrm{C}\right)}{m_{P}} \quad \text { Ec. } 4
$$

\subsection{Evaluación de la eficiencia del pykrete con sistema refrigerante en comparación con el gel comercial}

En una caja isotérmica se depositó una masa determinada de pykrete o gel congelado y se registró la temperatura del interior de la caja isotérmica en función del tiempo.

\section{Resultados y discusión}

Se evaluaron tres materiales celulósicos, aserrín, cascarilla de arroz entera y cascarilla de arroz molida.

Para identificar la mejor relación agua-material celulósico por cada material se prepararon tres pykretes con rela- ciones másicas diferentes $(0.05,0.14$ y 0.20$)$ y se congelaron por 48 horas, posteriormente se expusieron a temperatura ambiente y se evaluó el cambio de estado en función del tiempo (120 minutos) (Figura 1), en comparación del agua pura. Los tres materiales mostraron un comportamiento similar, siendo las relaciones másicas 0.14 y 0.20 las más prometedoras debido a que mantienen su estructura y parte del agua congelada en su interior, sin embargo el pykrete con relación másica de 0.20 presenta una resequedad excesiva por lo que concluye que el pykrete-material celulósico con relación másica de 0.14 es el más adecuado; el pykrete con la relación másica de 0.05 se comporta similar al agua pura debido posiblemente a que el material celulósico actúa como aislante térmico y este al tener una menor concentración pierde energía a una mayor velocidad. Una vez determinada que la relación másica agua-material 
celulósico más adecuada es la de 0.14 , se procede a la calibración del calorímetro de Joule determinando la capacidad calorífica del mismo de acuerdo a la ecuación 2.

Se realizaron tres replicas (Tabla 1 y 2), depositando en el vaso del calorímetro una masa determinada de agua y permitiendo que la temperatura del calorímetro y al agua agregada se equilibre, posteriormente se agrega agua a una temperatura menor a la del calorímetro y se agita constantemente hasta que la temperatura de los dos sistemas se equilibre. Para determinar la temperatura de equilibrio se registra el cambio de temperatura en función del tiempo hasta que la temperatura permanezca constante (Figura 2).

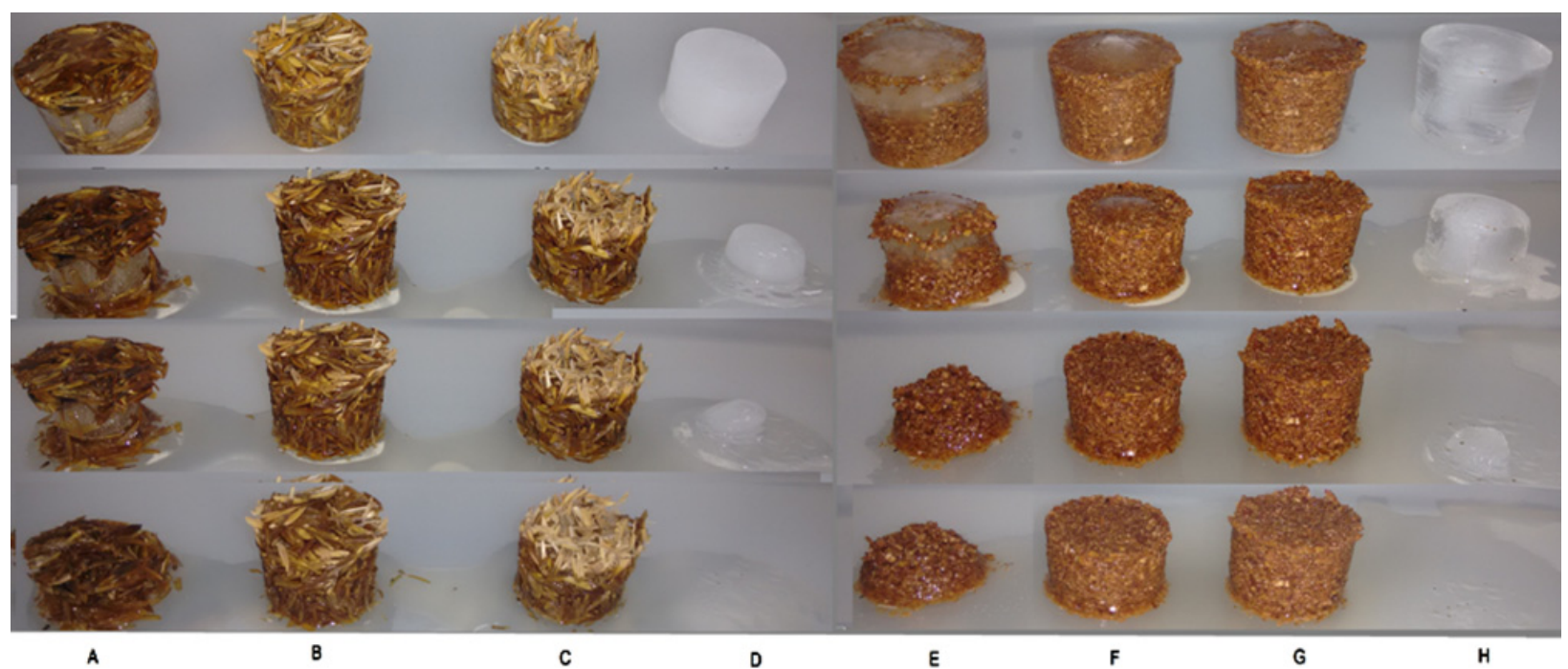

Figura 1. Cambio de estado de diferentes Pykretes a temperatura ambiente $\left(28^{\circ} \mathrm{C}\right)(\mathrm{A}=$ cascarilla de arroz $5 \%, \mathrm{~B}=$ cascarilla de arroz $14 \%, \mathrm{C}=$ cascarilla de arroz $20 \%$, D y H=Agua, E=Aserrín 5\%, F=Aserrín 14\%, G=Aserrín 20\%.

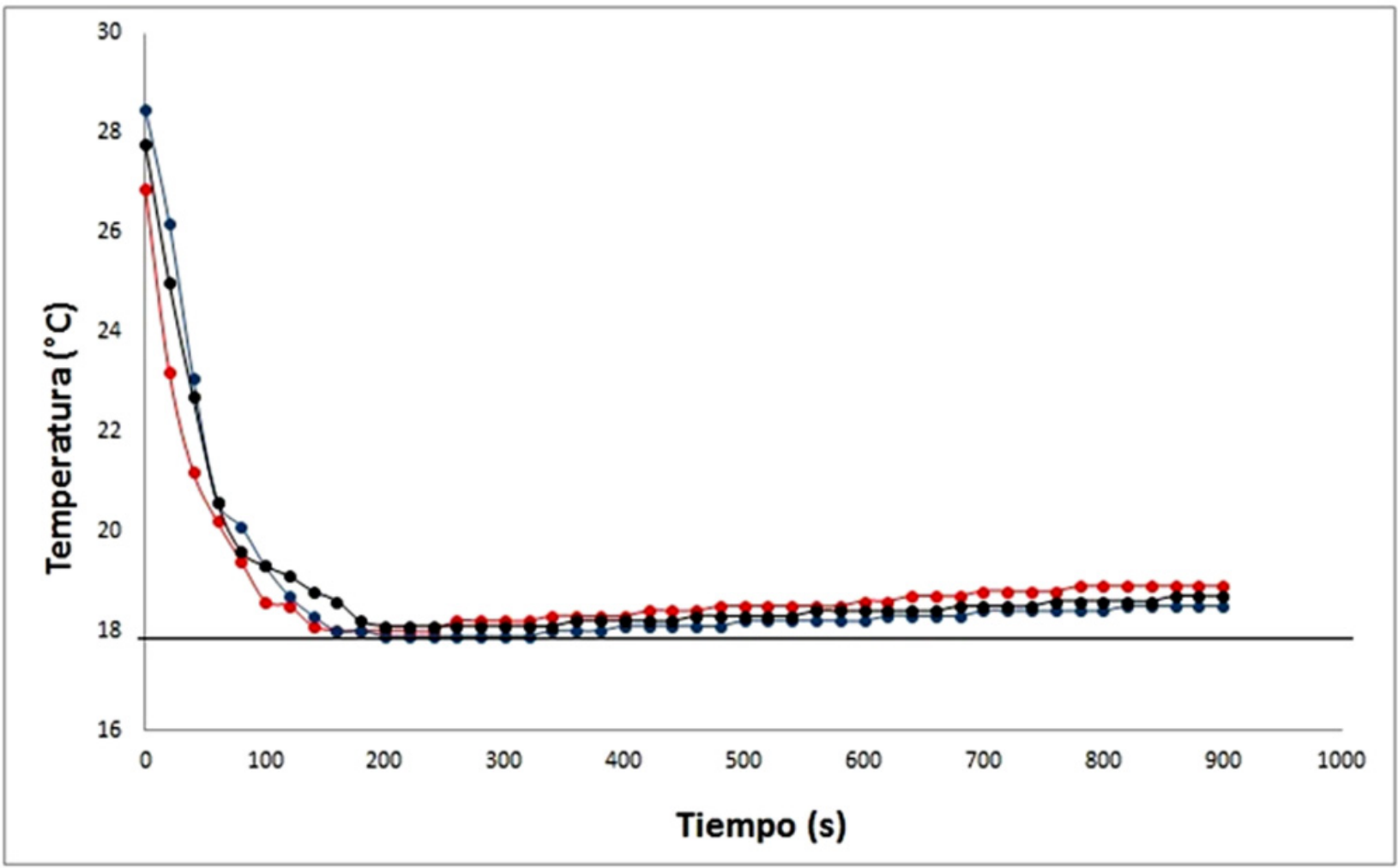

Figura 2. Curvas del cambio de la temperatura en función del tiempo del sistema agua-calorímetro para determinar la temperatura de equilibrio. 
Tabla 1. Calibración de calorímetro de Joule

\begin{tabular}{|c|c|c|c|}
\hline Replica & Material & Masa (g) & Temperatura (K) \\
\hline \multirow{4}{*}{1} & Calorímetro & 314.377 & 25.3 \\
\hline & Agua $T_{2}$ & 74.878 & 25.3 \\
\hline & Agua $T_{1}$ & 88.187 & 10.0 \\
\hline & Temperatura de equilibrio & & 17.6 \\
\hline \multirow{4}{*}{2} & Calorímetro & 314.377 & 25.3 \\
\hline & Agua $T_{2}$ & 105.724 & 25.3 \\
\hline & Agua $T_{1}$ & 103.620 & 10.6 \\
\hline & Temperatura de equilibrio & & 18.4 \\
\hline \multirow{4}{*}{3} & Calorímetro & 314.377 & 26.8 \\
\hline & Agua $\mathrm{T}_{2}$ & 105.083 & 26.8 \\
\hline & Agua $T_{1}$ & 100.708 & 10.0 \\
\hline & Temperatura de equilibrio & & 19.0 \\
\hline
\end{tabular}

Tabla 2. Capacidad calorífica aparente del calorímetro de Joule.

\begin{tabular}{|c|c|}
\hline Replica & $\begin{array}{c}\text { Capacidad calorífica aparente } \\
\left(\mathrm{Cal} \mathrm{g}^{-1}{ }^{\circ} \mathrm{C}^{-1}\right)\end{array}$ \\
\hline 1 & 0.0387 \\
\hline 2 & 0.0363 \\
\hline 3 & 0.0354 \\
\hline Promedio & $0.0368 \pm 0.0014$ \\
\hline$\%$ Coeficiente de variación & 3.81 \\
\hline
\end{tabular}

Se determinó que la capacidad calorífica aparente del calorímetro de Joule es de $0.0368 \pm 0.0014 \mathrm{cal} \mathrm{g}^{-1}{ }^{\circ} \mathrm{C}^{-1}$, con coeficiente de variación porcentual de 3.81. Una vez calibrado el calorímetro de procedió a determinar el calor latente de fusión aparente de los tres pykretes (agua-aserrín, agua-cascarilla de arroz entera y aguacascarilla de arroz molida) del gel comercial de acuerdo a la ecuación 4. Para determinar la temperatura de equilibrio se procedió de igual forma que para calibrar el calorímetro de Joule (Figura 2).

En las tablas 3-5, se presentan los diferentes datos para calcular el calor latente de fusión aparente de los tres

Tabla 3. Calor latente de fusión aparente del pykrete agua-cascarilla de arroz entera.

\begin{tabular}{|c|c|c|c|c|c|c|}
\hline Replica & $\begin{array}{l}\text { Masa (g) } \\
\text { de } \\
\text { Pykrete }\end{array}$ & $\begin{array}{c}\text { Porcentaje } \\
\text { P/P de } \\
\text { cascarilla de } \\
\text { arroz entera }\end{array}$ & $\begin{array}{c}\text { Masa de } \\
\text { agua en el } \\
\text { calorímetro }\end{array}$ & $\begin{array}{l}\text { Temperatura del } \\
\text { sistema Agua- } \\
\text { calorímetro }\left({ }^{\circ} \mathrm{C}\right)\end{array}$ & $\begin{array}{l}\text { Temperatura } \\
\text { de equilibrio } \\
\left({ }^{\circ} \mathrm{C}\right)\end{array}$ & $\begin{array}{l}\text { Calor latente de } \\
\text { fusión aparente } \\
\text { (cal/g) }\end{array}$ \\
\hline 1 & 23.662 & 5 & 186.502 & 28.5 & 17.9 & 71.2 \\
\hline 2 & 23.729 & 5 & 189.412 & 28.1 & 17.8 & 69.8 \\
\hline 3 & 22.223 & 5 & 189.909 & 27.3 & 17.9 & 67.7 \\
\hline & & & & & Promedio & $69.6 \pm 1.8$ \\
\hline \multicolumn{6}{|c|}{$\%$ Coeficiente de Variación } & 2.1 \\
\hline 1 & 18.247 & 14 & 194.927 & 27.0 & 19.7 & 64.0 \\
\hline 2 & 17.781 & 14 & 191.106 & 30.2 & 23.0 & 60.4 \\
\hline 3 & 18.751 & 14 & 188.855 & 30.0 & 22.3 & 61.2 \\
\hline \multicolumn{6}{|c|}{ Promedio } & $61.9 \pm 1.9$ \\
\hline \multicolumn{6}{|c|}{ \% Coeficiente de Variación } & 2.5 \\
\hline 1 & 15.846 & 20 & 193.199 & 29.7 & 23.4 & 59.9 \\
\hline 2 & 15.301 & 20 & 186.154 & 28.7 & 22.6 & 58.0 \\
\hline 3 & 15.535 & 20 & 193.338 & 29.1 & 23.1 & 57.9 \\
\hline \multicolumn{6}{|c|}{ Promedio } & $58.8 \pm 1.1$ \\
\hline \multicolumn{6}{|c|}{$\%$ Coeficiente de Variación } & 1.5 \\
\hline
\end{tabular}


Tabla 4. Calor latente de fusión aparente del pykrete agua-cascarilla de arroz molida.

\begin{tabular}{|c|c|c|c|c|c|c|}
\hline Replica & $\begin{array}{l}\text { Masa (g) } \\
\text { de } \\
\text { Pykrete }\end{array}$ & $\begin{array}{c}\text { Porcentaje } \\
\text { P/P de } \\
\text { cascarilla de } \\
\text { arroz molida }\end{array}$ & $\begin{array}{l}\text { Masa de } \\
\text { agua en el } \\
\text { calorímetro }\end{array}$ & $\begin{array}{l}\text { Temperatura del } \\
\text { sistema Agua- } \\
\text { calorímetro }\left({ }^{\circ} \mathrm{C}\right)\end{array}$ & $\begin{array}{l}\text { Temperatura } \\
\text { de equilibrio } \\
\left({ }^{\circ} \mathrm{C}\right)\end{array}$ & $\begin{array}{l}\text { Calor latente de } \\
\text { fusión aparente } \\
\text { (cal/g) }\end{array}$ \\
\hline 1 & 24.346 & 5 & 193.025 & 28.7 & 18.0 & 72.3 \\
\hline 2 & 25.523 & 5 & 195.501 & 29.1 & 17.9 & 73.3 \\
\hline 3 & 22.703 & 5 & 189.035 & 28.4 & 18.1 & 73.3 \\
\hline \multicolumn{6}{|c|}{ Promedio } & $73.0 \pm 0.6$ \\
\hline \multicolumn{6}{|c|}{ \% Coeficiente de Variación } & 0.7 \\
\hline 1 & 18.529 & 14 & 189.261 & 27.7 & 20.0 & 64.6 \\
\hline 2 & 18.479 & 14 & 191.210 & 27.0 & 19.1 & 68.7 \\
\hline 3 & 18.508 & 14 & 184.564 & 28.1 & 20.2 & 64.6 \\
\hline \multicolumn{6}{|c|}{ Promedio } & $66.0 \pm 2.3$ \\
\hline \multicolumn{6}{|c|}{ \% Coeficiente de Variación } & 2,9 \\
\hline 1 & 20.020 & 20 & 189.790 & 28.1 & 20.5 & 57.6 \\
\hline 2 & 20.745 & 20 & 190.679 & 27.5 & 19.8 & 56.9 \\
\hline 3 & 20.796 & 20 & 191.358 & 27.7 & 20.0 & 56.7 \\
\hline \multicolumn{6}{|c|}{ Promedio } & $57.1 \pm 0.5$ \\
\hline \multicolumn{6}{|c|}{$\%$ Coeficiente de Variación } & 0.7 \\
\hline
\end{tabular}

Tabla 5. Calor latente de fusión aparente del pykrete agua-aserrín.

\begin{tabular}{|c|c|c|c|c|c|c|}
\hline Replica & $\begin{array}{l}\text { Masa (g) } \\
\text { de } \\
\text { Pykrete }\end{array}$ & $\begin{array}{l}\text { Porcentaje } \\
\text { P/P de } \\
\text { aserrín }\end{array}$ & $\begin{array}{c}\text { Masa de } \\
\text { agua en el } \\
\text { calorímetro }\end{array}$ & $\begin{array}{l}\text { Temperatura del } \\
\text { sistema Agua- } \\
\text { calorímetro }\left({ }^{\circ} \mathrm{C}\right)\end{array}$ & $\begin{array}{c}\text { Temperatura } \\
\text { de equilibrio } \\
\left({ }^{\circ} \mathbf{C}\right)\end{array}$ & $\begin{array}{l}\text { Calor latente de } \\
\text { fusión aparente } \\
\text { (cal/g) }\end{array}$ \\
\hline 1 & 23.872 & 5 & 190.393 & 29.8 & 18.9 & 73.7 \\
\hline 2 & 26.177 & 5 & 184.567 & 30.0 & 18.0 & 72.3 \\
\hline 3 & 25.538 & 5 & 186.172 & 28.3 & 17.0 & 70.8 \\
\hline \multicolumn{6}{|c|}{ Promedio } & $72.3 \pm 1.4$ \\
\hline \multicolumn{6}{|c|}{ \% Coeficiente de Variación } & 1.6 \\
\hline 1 & 19.620 & 14 & 190.505 & 28.7 & 21.0 & 59.5 \\
\hline 2 & 19.887 & 14 & 192.352 & 28.8 & 20.9 & 61.3 \\
\hline 3 & 19.850 & 14 & 194.529 & 28.6 & 20.9 & 60.2 \\
\hline \multicolumn{6}{|c|}{ Promedio } & $60.3 \pm 0.9$ \\
\hline \multicolumn{6}{|c|}{ \% Coeficiente de Variación } & 1.2 \\
\hline 1 & 20.047 & 20 & 192.740 & 27.5 & 20.7 & 50.3 \\
\hline 2 & 19.854 & 20 & 192.560 & 27.3 & 20.5 & 51.1 \\
\hline 3 & 20.526 & 20 & 193.382 & 29.2 & 21.9 & 52.7 \\
\hline \multirow{2}{*}{\multicolumn{6}{|c|}{$\frac{\text { Promedio }}{\text { iciente de Varia }}$}} & $51.4 \pm 1.3$ \\
\hline & & & & & & 2.0 \\
\hline
\end{tabular}

pykretes (agua-cascarilla de arroz entera, agua-cascarilla de arroz molida y agua-aserrín), calculados de acuerdo a la ecuación 4 , la cual asume que la temperatura inicial del pykrete es de cero grado centígrados y que una vez se funda la totalidad del agua se libera material celulósico y agua a cero grados centígrados tomando como calor específico para el material celulósico el de la madera de $0.6 \mathrm{cal} \mathrm{g}^{-1}{ }^{\circ} \mathrm{C}^{-1}($ Lide, 2003$)$

En todos los casos, se observa que al aumentar el porcentaje de material celulósico el calor latente de fu- sión aparente disminuye debido posiblemente a que al reducir el porcentaje de agua en el pykrete la energía necesaria para fundir el agua dentro del material es menor. En este sentido, la capacidad de refrigeración del pykrete, depende del porcentaje de agua del material, pero a su vez la velocidad de fusión del sistema el cual se ve afectada por la cantidad de material celulósico presente en el pykrete, por lo que una disminución de aserrín o cascarilla de arroz en el mismo permitiría una mejor refrigeración pero disminuiría el tiempo de vida útil del pykrete. 
En la tabla 6, se presenta el calor latente de fusión aparente del gel comercial, en donde el porcentaje de polímero en el gel comercial fue determinado por diferencia masa al secar una cantidad determinada del material dando aproximadamente $1.87 \pm 0.02 \%$, por lo que posiblemente el gel este compuesto por un polímero súper absorbente, por tanto su capacidad de refrigeración está relacionada con el alto porcentaje de agua, lo que permitiría una mejor refrigeración y a su vez actuaría como un aislante térmico lo que permitía al gel comercial descongelarse a una velocidad menor a la del hielo. La capacidad calorífica del polímero fue tomada como $0.2 \mathrm{cal} \mathrm{g}^{-1}{ }^{\circ} \mathrm{C}^{-1}$ (Lide, 2003).

Los resultados del calor latente de fusión aparente tanto del gel como de los diferentes pykretes, se presentan en la tabla 7. Mediante un análisis de varianza (ANOVA) (Delgado, et al., 2013), la homoscedasticidad se demuestra aplicando la prueba $\mathrm{G}$ del test de Cochran, donde $\mathrm{G}_{\text {Exp }}=0.181$ es menor al $\mathrm{G}_{\mathrm{Tab}}(\mathrm{a}=0,05$, $\mathrm{K}=10, \mathrm{n}=3)=0,445$ lo que significa que las variancias para los diferentes niveles de concentración de material celulósico y tipo de material son homogéneas, mostrando así que el nivel de concentración o tipo de material no influye en la variabilidad de los resultados, por lo tanto no se presenta una relación estadísticamente significativa entre la variable explicativa (concentración y tipo de material) y el error experimental. En cuanto al análisis de varianza (Tabla 8), mediante el test $\mathrm{F}$, el $\mathrm{F}_{\mathrm{Exp}}=72.65$ es mayor a $\mathrm{F}_{\mathrm{Tab}}=2.393$ con 9 grado de libertad en el numerador y con 20 grados de libertad en el denominador y con un nivel de significa-

Tabla 6. Calor latente de fusión aparente del gel comercial.

\begin{tabular}{r|c|c|c|r|r|r}
\hline Replica & $\begin{array}{c}\text { Masa (g) } \\
\text { de } \\
\text { Pykrete }\end{array}$ & $\begin{array}{c}\text { Porcentaje } \\
\text { P/P de } \\
\text { polimero }\end{array}$ & $\begin{array}{c}\text { Masa de } \\
\text { agua en el } \\
\text { calorímetro }\end{array}$ & $\begin{array}{c}\text { Temperatura del } \\
\text { sistema Agua- } \\
\text { calorímetro }\left({ }^{\circ} \mathbf{C}\right)\end{array}$ & $\begin{array}{c}\text { Temperatura } \\
\text { de equilibrio } \\
\left({ }^{\circ} \mathbf{C}\right)\end{array}$ & $\begin{array}{c}\text { Calor latente de } \\
\text { fusión aparente } \\
(\mathbf{c a l} / \mathbf{g})\end{array}$ \\
\hline 1 & 23.872 & 1.90 & 197.346 & 29.4 & 18.7 & 66.9 \\
\hline 2 & 26.177 & 1.85 & 189.251 & 27.6 & 17.1 & 66.2 \\
\hline 3 & 25.538 & 1.87 & 189.956 & 27.6 & 16.8 & 64.4 \\
\hline \multicolumn{7}{c|}{ Promedio } \\
\hline \multicolumn{7}{c}{ \% Coeficiente de Variación } \\
\hline
\end{tabular}

Tabla 7. Resultados del calor latente de fusión aparente de gel comercial y los diferentes Pykretes (W-CA5\%= Pykrete agua-cascarilla de arroz entera; W-CAM= Pykrete agua-cascarilla de arroz molida y W-A= Pykrete agua-aserrín).

\begin{tabular}{c|c|c|c|c|c|c|c|c|c|c}
\hline Resultados & Gel & $\begin{array}{c}\text { W-CA } \\
\mathbf{5 \%}\end{array}$ & $\begin{array}{c}\text { W-CA1 } \\
\mathbf{4 \%}\end{array}$ & $\begin{array}{c}\text { W-CA } \\
\mathbf{2 0} \%\end{array}$ & $\begin{array}{c}\text { W-CAM } \\
\mathbf{5 \%}\end{array}$ & $\begin{array}{c}\text { W-CAM } \\
\mathbf{1 4 \%}\end{array}$ & $\begin{array}{c}\text { W-CAM } \\
\mathbf{2 0} \%\end{array}$ & $\begin{array}{c}\text { W-A } \\
\mathbf{5 \%}\end{array}$ & $\begin{array}{c}\text { W-A } \\
\mathbf{4 \%}\end{array}$ & $\begin{array}{c}\text { W-A } \\
\mathbf{2 0} \%\end{array}$ \\
\hline $\mathbf{1}$ & 66.9 & 71.2 & 64.0 & 59.9 & 72.3 & 64.6 & 57.6 & 73.7 & 59.5 & 50.3 \\
\hline $\mathbf{2}$ & 66.2 & 69.8 & 60.4 & 58.0 & 73.3 & 68.7 & 56.9 & 72.3 & 61.3 & 51.1 \\
\hline $\mathbf{3}$ & 64.4 & 67.7 & 61.2 & 57.9 & 73.3 & 64.6 & 56.7 & 70.8 & 60.2 & 52.7 \\
\hline Suma & 197.5 & 208.7 & 185.6 & 175.8 & 218.9 & 197.9 & 171.2 & 216.8 & 181 & 154.1 \\
\hline$\overline{x_{k}}$ & 65.8 & 69.6 & 61.9 & 58.6 & 73.0 & 66.0 & 57.1 & 72.3 & 60.3 & 51.4 \\
\hline$n_{k}$ & 3 & 3 & 3 & 3 & 3 & 3 & 3 & 3 & 3 & 3 \\
\hline
\end{tabular}

Media del total de resultado, $x_{k}=63.58$

Número total de resultados, $\mathrm{N}=30$

Tabla 8 Tabla ANOVA para los resultados de la Tabla 7.

\begin{tabular}{c|c|c|c|c}
\hline Fuente & Suma de Cuadrados & Grados de Libertada & Cuadrado Medio & F Exp \\
\hline Entre sistemas & 1320.1 & 9 & 146.68 & 72.65 \\
\hline Intra sistemas & 40.4 & 20 & 2.02 & \\
\hline Total & 1360.5 & 29 & 46.91 & \\
\hline
\end{tabular}


ción $\alpha=0.05$, indicando que al menos el calor latente de fusión aparente de uno de los sistemas es estadísticamente significativa del resto, consolidando lo anteriormente mencionado respecto a que el porcentaje de material celulósico y el tipo de material influyen en el calor latente de fusión aparente de los sistemas (pykretes).

Ahora bien, debido a que el gel comercial es utilizado en la conservación de la cadena y la finalidad del presente trabajo es evaluar las propiedades termofísicas de diferentes pykretes a fin de reemplazar este material no biodegradable por sistemas orgánicos que sí lo son. Por ende se realizó un análisis de varianza comparando los resultados de cada pykrete con los del gel comercial (Tabla 8).

Tabla 9. Valores de $\mathrm{F}_{\text {Exp }}$ del ANOVA al comparar los resultados de cada uno de los 9 pykretes con los resultados del gel comercial.

\begin{tabular}{lc}
\hline Sistema & Valor de $\boldsymbol{F}_{\text {Exp }}$ \\
\hline Pykrete W-CA 5\% & 51.4 \\
Pykrete W-CA 14\% & 9.2 \\
Pykrete W-CA 20\%P & 61.2 \\
Pykrete W-CAM 5\% & 279.8 \\
Pykrete W-CAM 14\% & 8.9 \\
Pykrete W-CAM 20\% & 151.2 \\
Pykrete W-A 5\% & 128.2 \\
Pykrete W-A 14\% & 37.7 \\
Pykrete W-A 20\% & 293.3 \\
\hline
\end{tabular}

De acuerdo al test $\mathrm{F}$, el $\mathrm{F}_{\text {Exp }}$ es mayor en todos los casos al $\mathrm{F}_{\mathrm{Tab}}=7.709$ con 1 grado de libertad en el numerador, 4 grados de libertad en el denominador y con un nivel de significación $\alpha=0.05$., indicando que al menos el calor latente de fusión aparente de uno de los sistemas es estadísticamente significativa del resto. Si bien ningún sistema agua-material celulósico tiene propiedades termofisicas estadísticamente similares a la del gel comercial, los pykretes con una relación másica de 0.14 son los sistemas más similares al gel comercial, por lo que podrían ser utilizados como "dispositivos acumuladores de frío" y en particular el pykrete de cascarilla de arroz molida con fracción másica de 0.14 , es la mejor relación agua-material celulósico debido a que tiene un calor latente de fusión alto lo que lo transforma en un buen dispositivo refrigerante y a su vez la cantidad cascarilla de arroz es suficiente para permitir que el material permanezca congelado por un lapso de tiempo mayor a la del hielo.

Finalmente con el ánimo de evaluar la eficiencia del pykrete en comparación con el gel comercial, se registró el cambio de temperatura dentro del interior de una caja isotérmica al depositar $500 \mathrm{~g}$ de pykrete con una relación másica de material celulósico de 0.14 o gel comercial (Figura 3).

Se registró el cambio de temperatura durante aproximadamente 4 horas demostrando que los tres pykretes evaluados tienen una eficiencia muy similar a la del gel comercial, la tendencia del pykrete de cascarilla de arroz

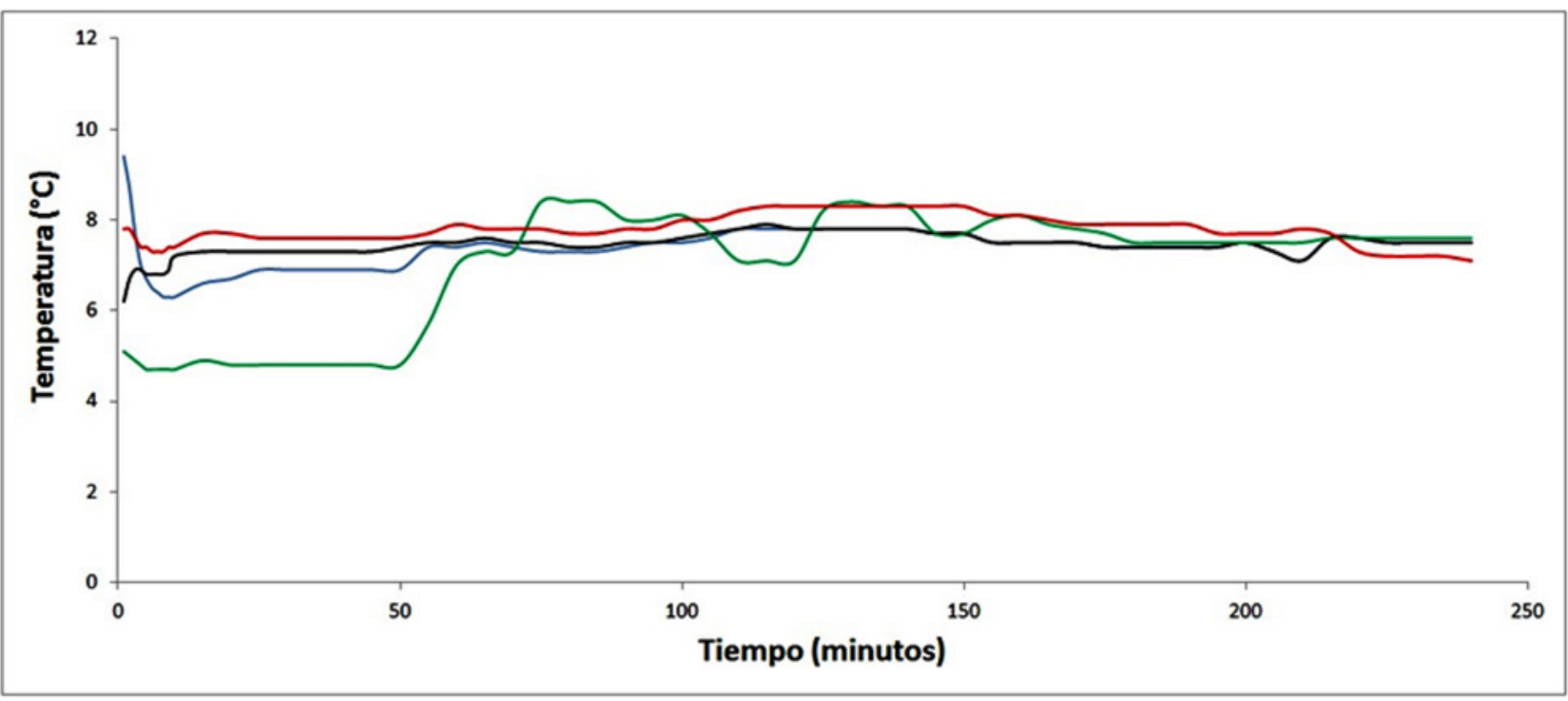

Figura 3. Cambio de temperatura de una caja isotérmica en función del tiempo, al depositar 500 g de Pykrete (14\% de material celulósico) o gel comercial. $(\mathrm{Gel}=$ línea azul; Cascarilla de arroz= línea negra; Cascarilla de arroz molida= línea verde y Aserrín= línea roja). 
entera prácticamente se solapa con la tendencia del gel comercial, y los pykretes de cascarilla de arroz molida y de aserrín si bien presentan distorsiones, mantienen la temperatura del sistema por debajo de $10^{\circ} \mathrm{C}$.

\section{Conclusiones}

A medida que se aumenta el porcentaje de material vegetal en el sistema la velocidad de fusión disminuye por lo que los sistemas con 14 y $20 \%$ son los mejores sistemas, ahora de acuerdo al calor latente de fusión aparente de cada sistema, este claramente depende la proporción de material celulósico, disminuyendo a medida que el porcentaje de aserrín o cascarilla incrementa, por tanto, el material celulósico actúa como un aislante térmico manteniendo durante un mayor tiempo el sistema congelado.

Al evaluar el cambio de temperatura en función del tiempo de un sistema caja isotérmica + pykrete o caja isotérmica + gel comercial, los pykretes de cascarilla de arroz molida y entera presentan comportamientos similares a los del gel comercial, mostrando una eficiencia similar a los dispositivos compuestos por gel.

Finalmente a partir los resultados del ANOVA se puede decir que el pykrete de cascarilla de arroz molida en una relación másica de 0.14 podría ser utilizado como dispositivo de refrigeración debido a que sus propiedades termofísicas son similares a las del gel comercial, material que es utilizado para tal fin.

\section{Referencias bibliográficas}

Broekmeulen, R.A.C.M., 2001. Modelling the management of distribution centers L.M.M. Tijkskens, M.L.A.T.M. Hertog, B.M. NicolaR (Eds.), Food Process Modelling (third ed.), CRC Press, Washington, DC. pp. 432-448.

Browne, M., Allen, J., 1998. Logistics of food transport. R. Heap, M. Kierstan, G. Ford (Eds.), Food Transportation, Blackie Academic \& Professional, London. pp. 22-50.

Cambel, D.T., Stanley, J.C., 1963. Experimental and quasi-experimental designs for research. Houghton Mifflin Company, Dallas, pp. 1-76.

Cochran, W.G., 1950. The Comparison of Percentages in Matched Samples, Biometrika., 37, pp. 256-266.
Delgado, D.R., Rodríquez, G.A., Martínez, J.A., Rojas, J.H., Martínez, F., 2013. Validación de una metodología analítica empleando espectrofotometría ultravioleta para el estudio de la solubilidad de algunas sulfonamidas en mezclas cosolventes alcohol + agua. Rev.Colomb.Quim., 42(3), pp. 31-40.

Dubelaar, C., Chow, G., Larson, P., 2001. Relationships between inventory, sales and service in a retail chain store operation. Int. J. Phys. Distrib. Logist.Manag., 31, pp. 96-108.

Evans, J., 1992. Consumer handling of chilled foods perceptions and practice. Int. J. Refrigeration. 15, pp. 290-298.

Evans, J.A., Stanton, J.I., Russell, S.L., James, S.J., 1991. Consumer Handling of Chilled Foods: a Survey of Time and Temperature Conditions. MAFF Publications, London.

Giannakourou, M.C., Koutsoumanis, K., Nychas, G.J.E., Taoukis, P.S., 2001. Development and assessment of an intelligent shelf life decision system for quality optimization of the food chill chain. J. Food Prot., 64, pp. 1051-1057.

Giannakourou, M., Taoukis, P., 2003. Application of a TTI-based distribution management system for quality optimization of frozen vegetables at the consumer end. J. Food Sci., 68, pp. 201-209.

Giannakourou, M.C., Taoukis, P.S., 2003. Kinetics modelling of vitamin $\mathrm{C}$ loss on frozen green vegetables under variable storage conditions. Food Chem, 83, pp. 33-41.

Giannakourou, M.C., Taoukis, P.S., 2002. Systematic application of time temperature integrators as tools for control of frozen vegetable quality. J. Food Sci., 67, pp. 2221-2228.

Giannakourou, M.C., Taoukis, P.S., 2002. Systematic application of time temperature integrators as tools for control of frozen vegetable quality. J. Food Sci., 67, pp. 2221-2228.

James, S.J., Evans, J., 1992. Consumer handling of chilled foods - temperature performance. Int. J. Refrigeration. 15, pp. 299-30.

James, S.J., Evans, J., 1992. The temperature performance of domestic refrigerators. Int. J. Refrigeration, 15, pp. 313-319. 
Kennedy, J., Jackson, V., Blair, I.S., McDowell, D.A., Cowan, C., Bolton D.J., 2005, Food safety knowledge of consumers and the microbiological and temperature status of their refrigerators. J. Food Prot., 68, pp. 1421-1430.

Koutsoumanis, K., Giannakourou, M.C., Taoukis, P.S., Nychas, G.J.E., 2002. Application of shelf life decision system (SLDS) to marine cultured fish quality. Int. J. Food Microbiol., 73, pp. 375-382.

Koutsoumanis, K., Taoukis, P.S., Nychas, G.J.E., 2005. Development of a Safety Monitoring and Assurance System (SMAS) for chilled food products. Int. J. Food Microbiol., 100, pp. 253-260.

Levine, I.N., 2004. Fisicoquímica, McGraw Hill, Madrid, pp. 53-54.

Likar, L., Jevšnik, M., 2006. Cold chain maintaining in food trade. Food Control, 17, pp. 108-113.

Lide, D.R., 2003. CRC Handbook of Chemistry and Physics, New York.
Pronk, A.D.C., Vasiliev, N.K., Janssen, F.H.M.E., Sprayingpykrete, R.W.G., 2014. The spraying of water and wood fibers to reinforce ice Proceedings of the IASS-SLTE 2014 Symposium "Shells, Membranes and Spatial Structures:Footprints", Brasilia, Brazil.

Tijkens, L.M.M., Koster, A.C., Jonker, J.M.E., 2001. Concepts of chain management and chain optimisation. L.M.M. Tijkskens, M.L.A.T.M. Hertog, B.M. NicolaR (Eds.), Food Process Modelling (third ed.), CRC Press, Washington, DC. pp. $448-469$.

Vasilieva, N.K., Pronkb, A.D.C., Shatalinaa, I.N., Janssenb, F.H.M.E., Houbenb, R.W.G., 2015. A review on the development of reinforced ice for use as a building material in cold regions, Cold Regions Science and Technology., 115, pp. 56-63.

Wells, J.H., Singh, R.P., 1989. A quality-based inventory issue policy for perishable foods. J. Food Process. Preserv., 12, pp. 271-292. 